\title{
High-Efficiency, Two-Step Scarless-Markerless Genome Genetic Modification in Salmonella enterica
}

\author{
Shizhong Geng ${ }^{1} \cdot$ Qin Tian $^{1} \cdot$ Shuming An ${ }^{1} \cdot$ Zhiming Pan $^{1} \cdot$ Xiang Chen $^{1} \cdot$ \\ Xinan Jiao ${ }^{1}$
}

Received: 27 September 2015/ Accepted: 26 December 2015/Published online: 16 February 2016

(C) The Author(s) 2016. This article is published with open access at Springerlink.com

\begin{abstract}
We present a two-step method for scarlessmarkerless genome genetic modification in Salmonella enterica based on the improved suicide plasmid pGMB 152. The whole LacZYA gene can provide a lacZ-based blue/ white screening strategy for fast selection of doublecrossover mutants by allelic exchange. The high efficiency of this genetic engineering strategy permits the study of gene function by gene knockin, site-directed mutagenesis, and gene knockout to construct live attenuated vaccines.
\end{abstract}

\section{Introduction}

Genome genetic modification, including gene knockout, gene knockin, and site-directed mutagenesis by replacing target genes with in vitro-modified alleles is an essential tool for studying the genetic basis of bacterial phenotypes at the molecular level $[4,17]$. Current methods are largely based on the principles of allelic exchange using a conventional suicide plasmid, such as pGMB151 [8, 11]. pGMB151 is a derivative of the suicide plasmid pKNG101 [11] and includes oriR6K, RP4, sacBR, insB, and $\operatorname{str} A B$ with the bla gene from plasmid pBR322 cloned at the Xba I site. It is a suicide plasmid because of its requirement for the pir protein, which is supplied by $\lambda$ pir in certain strains of Escherichia coli, such as SM10 [18].

Xinan Jiao

jiao@yzu.edu.cn

1 Jiangsu Key Laboratory of Zoonosis, Jiangsu Co-Innovation Center for Prevention and Control of Important Animal Infectious Diseases and Zoonoses, Yangzhou University, Yangzhou 225009, China
The current suicide plasmid method may not be very efficient because of low recombination rates or illegitimate recombination, so they are often improved [12, 17]. To improve the efficiency of selecting precise gene-deletion mutants and simplifying the process of genome genetic modification, the $s a c B$ gene was introduced into a suicide plasmid for a kind of counterselection. The $s a c B$ gene in the suicide plasmid encodes levansucrase, which hydrolyzes sucrose and glucose to fructose, resulting in the production of a toxic fructan that kills the recombinants containing the suicide plasmid. Thus, sucrose is often used to screen bacteria that have lost the suicide plasmid after a double-crossover event in a single-crossover recombinant. Loss of the suicide plasmid can be easily verified via loss of vector-encoded antibiotic resistance. However, this kind of sucrose counterselection only offers one possibility, and its susceptibility to sucrose is variable in different kinds of gram-negative bacteria: for example, in Salmonella enterica, most single-crossover recombinant bacteria show resistance to sucrose, and a few single-crossover recombinant bacteria are sensitive to sucrose $[7,16]$.

Additionally, antibiotic resistance genes have been used to replace the target gene as selectable markers of successful gene deletion $[8,14,15,17]$. Although some enzymes may also remove the antibiotic resistance gene, its flanking sequences may not be completely removed. This is an undesirable situation, particularly when precise and scarless-markerless genome genetic modifications are required, such as in genetically engineered attenuated vaccines.

A precise and scarless-markerless genome genetic modification, such as gene knockout for attenuated Salmonella vaccines, gene knockin, and site-directed mutagenesis are more difficult without any exogenous nucleotide residuals. The main problem is that double- 
crossover events following the loss of a suicide plasmid are very difficult to distinguish because of the low frequency of allelic exchange and resistance to sucrose. We believe that a suicide plasmid should have another visual biomarker as a counter selectable tool. Thus, in this study, to improve the efficiency of allelic exchange for genome genetic modification, a novel pGMB152 vector was constructed with the lacZYA operon incorporated into pGMB151 to provide LacZ-based blue-white selection [1]. The LacZ gene, a well-known reporter gene expressing $\beta$-galactosidase, can provide blue-white selection to make the existence of molecular cloning visible $[2,6,13]$ and could be used as a biomarker for the suicide plasmid. Bacteria colonies with pGMB152 will show as blue and those without pGMB152 will show as white; therefore, bacterial colonies with different colors could be distinguished easily during the different processes of genome genetic modification. The new plasmid was used to knock out the $h s d M$ gene [9] in Salmonella pullorum to construct a candidate-attenuated Salmonella vaccine, to repair pseudogene $f h A$ by knockin and pseudogene $f l g k$ by site-directed mutagenesis.

\section{Materials and Methods}

\section{Strains, Plasmids, and Media}

The strains and plasmids used in this study are listed in Table 1. Strains were grown routinely in rich liquid or solid Luria Broth media (LB). The media was supplemented with kanamycin $(50 \mu \mathrm{g} / \mathrm{mL})$, ampicillin $(100 \mu \mathrm{g} / \mathrm{mL})$, and streptomycin $(25 \mu \mathrm{g} / \mathrm{mL})$ as required. Solid LB media with $10 \%$ sucrose and without $\mathrm{NaCl}$ was used to screen for gene-deletion mutants during the process of allelic exchange.

\section{Construction of Suicide Plasmid pGMB152}

The lacZYA cassette was excised from pFUSE (Deng et al. [4]) using restriction endonucleases Sam I and Sal I and ligated into pGMB151 digested with the same restriction recognition sites. This novel plasmid was named pGMB152.

\section{Two-Step Scarless-Markerless Knockout of hsdM Gene}

The chloramphenicol resistance gene $\left(\mathrm{Cm}^{R}\right)$ from plasmid pKD3, with $54 \mathrm{bp}$ of homologous sequences of the $h s d M$ gene flanking the two ends of $\mathrm{Cm}^{R}$, was PCR amplified using primers $h s d M-\lambda-\mathrm{F} / \mathrm{R}$. This fragment was transformed into S. pullorum S06004 to inactivate the $h s d M$ gene, with the help of plasmid pKD46 by the $\lambda$-red method, to construct S. Pullorum hsdM::Cm${ }^{R}$ with an antibiotic marker [3].

An in vitro-modified allele was constructed into pGMB152. Two DNA fragments (1083-bp $h s d M 12$ and 1103-bp $h s d M 34)$ flanking the $h s d M$ gene were amplified by PCR using the primers shown in Table 2 . The in vitromodified allele of $h s d M$ 12-34 ( $\Delta h s d M)$ was amplified using splicing by overlapping Extension PCR using primers $h s d M 12-\mathrm{F}$ and $h s d M 34-\mathrm{R}$, because the primer $h s d M$ $34-\mathrm{F}$ was the reverse complement of the primer $h s d M 12-\mathrm{R}$ [5]. The amplified fragment was sub-cloned into the Sal I site of pMD20-T. A kanamycin-resistant gene cassette $\left(K m^{R}\right)$ was inserted at the Xho I in the middle of the fragment, with a $\mathrm{Sal}$ I site at each end [3, 8]. Subsequently, the DNA fragment between the two Sal I sites in the plasmid pMD20T- $\Delta h s d M$ was introduced into the Sal I cloning site of suicide plasmid pGMB152 to construct recombinant suicide plasmid pGMB152- $\Delta h s d M$.

\section{Blue-White Selection for White Double-Crossover from Blue Single-Crossover Bacteria}

Following a previous protocol [8], the plasmid pGMB152$\Delta h s d M$ was electroporated into $E$. coli $\chi 7213$, which is diaminopimelic acid (DAP) dependent and kanamycin resistant. Blue colonies were selected on LB agar with $40 \mu \mathrm{g} / \mathrm{ml}$ X-gal, $1 \%$ DAP, and three antibiotics
Table 1 Strains and plasmids used in this study

\begin{tabular}{lllll}
\hline Material & Name & Use & Source & Reference \\
\hline Bacteria & S.Pullorum S06004 & Recipient strain & Our laboratory & {$[9]$} \\
& E.coli. $\chi 7213$ & Donor strain & Gift from Dr. R. Curtiss III & {$[10]$} \\
& E.coli.spy372 & For cloning & Gift from Dr. R. Curtiss III & \\
& E.coli. DH5 $\alpha$ & For cloning & Takara company & \\
Plasmids & pGMB151 & Suicide plasmid & Our laboratory & \\
& pGMB152 & Suicide plasmid & This study & \\
& pFUSE & To offer LacZYA gene & Our laboratory \\
& pMD20-T & For cloning & Takara company & \\
\hline
\end{tabular}


Table 2 Primers used in this study (enzyme sites are shown in lower case)

\begin{tabular}{|c|c|c|c|c|}
\hline Fragments amplified & Primers & Primer sequences $\left(5^{\prime}-3^{\prime}\right)$ & Amplicon size (bp) & Note \\
\hline \multirow[t]{6}{*}{$h s d M-\lambda$} & $h s d M-\lambda$ & CTGCTGACCGAAATGCTCGAACC & & \\
\hline & & GTTCCAGGGCAAAATTTATGACC & & \\
\hline & & CCTGCTGCtgtgtaggctggagctgcttcg & & \\
\hline & $h s d M-\lambda$ & TCGGCTTCGCCGCTGGTGTTGGA & & \\
\hline & & GCTCATCGAGCCGTTCGCCAGCA & & \\
\hline & & CAAAACCGcatatgaatatcctccttag & & \\
\hline \multirow[t]{4}{*}{ hsdM12 } & $h s d M 12-\mathrm{F}$ & aagtcgacctcgagATGCGGGTTCGGTTT & 1083 & Sal I, Xho I \\
\hline & & GTTTG & & \\
\hline & $h s d M 12-\mathrm{R}$ & TTCCTGATTGAGTTCATCATGGCC & & \\
\hline & & TGAAAAGACAATCCCACTCAATG & & \\
\hline \multirow[t]{3}{*}{ hsdM34 } & $h s d M 34-\mathrm{F}$ & CATTGAGTGGGATTGTCTTTTCAG & 1103 & Sal I \\
\hline & & GCCATGATGAACTCAATCAGGAA & & \\
\hline & $h s d M 34-\mathrm{R}$ & aagtcgacTGGTCCGAGCCGGATAAATG & & \\
\hline \multirow[t]{2}{*}{$\Delta h s d M-Y Z$} & $\Delta h s d M-\mathrm{YZ}-\mathrm{F}$ & TGACCACCACCAATGCTACC & $1920(w t) 301(\Delta)$ & \\
\hline & $\Delta h s d M-\mathrm{YZ}-\mathrm{R}$ & GTGGAACTGCTGGATGTGGA & & \\
\hline \multirow[t]{6}{*}{$f h A-\lambda$} & $f l h A-\lambda-\mathrm{F}$ & СCTTTAATATTGCGCTATCGATCATG & & \\
\hline & & GTGCTGCTGGTGGCGATGTTTACCC & & \\
\hline & & AGAtgtgtaggctggagctgcttcg & & \\
\hline & $f l h A-\lambda-\mathrm{R}$ & CCAGCGCCGGGATCTGGGCGACCAG & & \\
\hline & & GCCGTCGCCAATGGTCAGCAGGGTG & & \\
\hline & & TAGCcatatgaatatcctccttag & & \\
\hline \multirow[t]{3}{*}{$\operatorname{flhA}(\mathrm{P} 125109)$} & flhA-F & AAgtcgac ctcgag CGGTAAGTCGCTACAGCCAA & 2349 & Sal I, Xho I \\
\hline & flhA-R & AAgtcgac agatct CCTGAG & & Sal I \\
\hline & & ACACGATCCAAC & & \\
\hline \multirow[t]{6}{*}{ flgK- $\lambda$} & flgK- $\lambda-F$ & TATTCTGGCGCAGGCAAACAGT & & \\
\hline & & ACGTTAGGGGCTGGCGGCTGGA & & \\
\hline & & TAGGTAATGGtgtgtaggctggagctgcttcg & & \\
\hline & flgK- $\lambda-\mathrm{R}$ & TGCGCCACGCTGGAGCCAATCGCGA & & \\
\hline & & TATTGACCTGTTTATCCTGATCGCGC & & \\
\hline & & AGAcatatgaatatcctccttag & & \\
\hline \multirow[t]{4}{*}{$\operatorname{flg} K(\mathrm{P} 125109)$} & $f \lg K-\mathrm{F}$ & AAcccggg CTCGAGGCTGAA & 2240 & Sma I, Xho I \\
\hline & & AAGTATGCGCGAGG & & \\
\hline & $\operatorname{flg} K-\mathrm{R}$ & cccggg AAGTTATCGCTGTCG & & Sma I \\
\hline & & CCGGTAT & & \\
\hline
\end{tabular}

(kanamycin, ampicillin, and streptomycin). Conjugation was then carried out between a donor of blue E. coli $\chi 7213$ (pGMB152- $\Delta h s d M$ ) and a recipient of $S$. Pullorum $h s d M: \because C m^{R}$. Equal volumes of $24-\mathrm{h}$ LB cultures from the donor and recipient were mixed and spotted immediately onto a nylon filter on an LB plate [8,9]. After $36 \mathrm{~h}$ of conjugation at $37^{\circ} \mathrm{C}$, the filter was washed with $10 \mathrm{mM}$ $\mathrm{MgSO}_{4}$ and the bacteria were plated onto LB plates containing $\mathrm{X}$-gal and the three antibiotics (kanamycin, ampicillin, and streptomycin). The blue colonies presumably resulted from a single-crossover event where the suicide plasmid pGMB152 was integrated into the chromosome at $h s d M$. A random single colony was purified and sub-cultured 4-5 times in liquid LB with $10 \%$ sucrose and without $\mathrm{NaCl}[7,16]$. Bacteria were then plated onto LB agar plates and screened for white colonies without chloramphenicol resistance to confirm the presence of plasmid excised double-crossover events. All white colonies were detected again with chloramphenicol selection: the doublecrossover deletants should be susceptible to chloramphenicol because $\mathrm{Cm}^{R}$ gene was replaced by in vitromodified allele. 
Fig. 1 Construction of an in vitro-modified allele
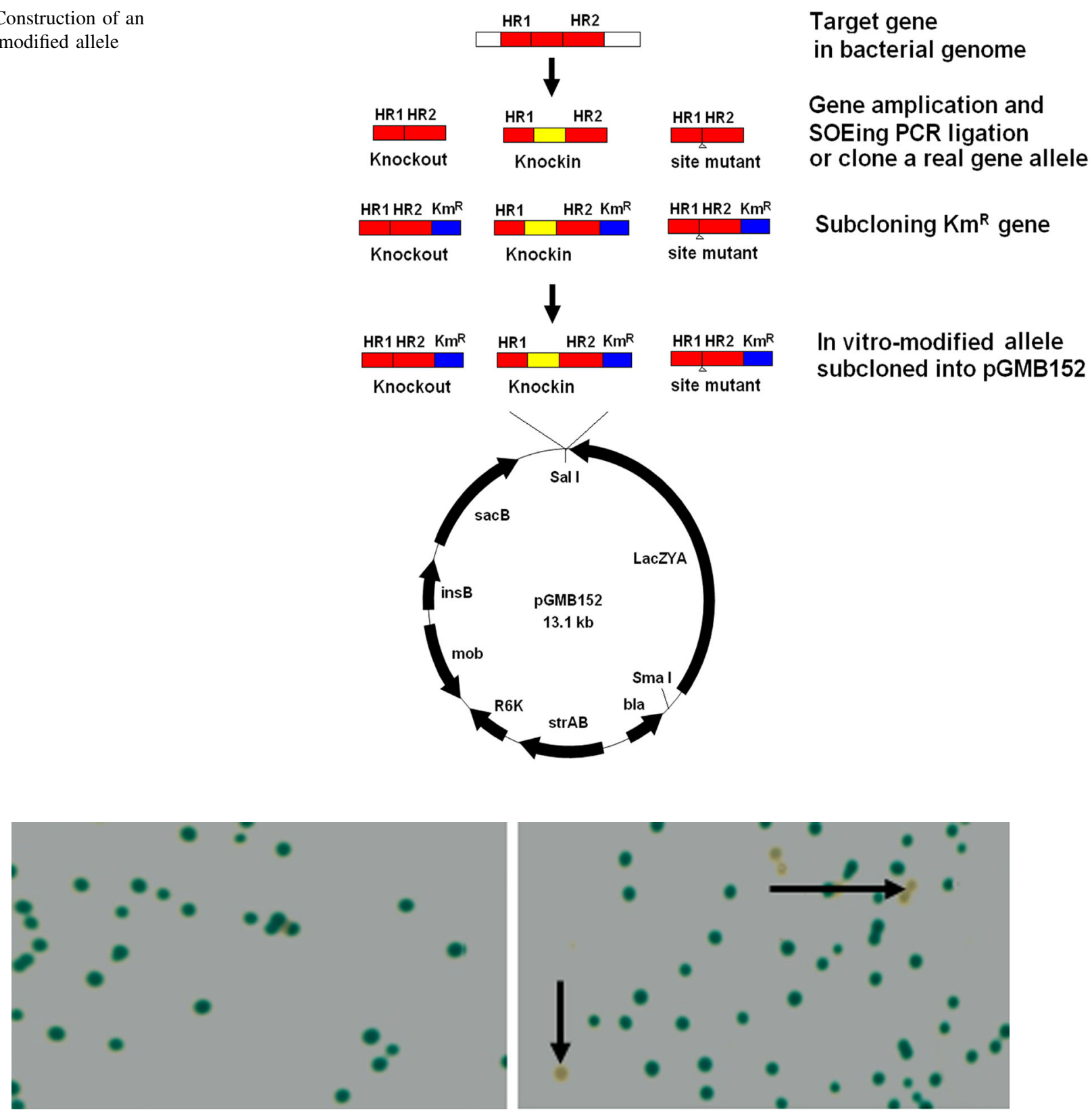

Fig. 2 Blue-white selection. Left-hand panel shows blue colonies resulting from a single-crossover event, and the right-hand panel shows the white colonies (possible $h s d M$ deletants) from a double-crossover event (example of $h s d M$ ) (Color figure online)

\section{Confirmation of Deletants by PCR}

The chromosomal duplication was segregated by homologous recombination between the flanking direct repeats, ultimately leaving one copy of the gene on the chromosome: either the wild-type copy or the deletant copy, and the suicide plasmid pGMB152 were lost simultaneously. The white colonies were further identified by PCR using primers $\Delta h s d M$-YZ-F and $\Delta h s d M$-YZ-R to distinguish the deletants from the wild-type bacteria.

\section{Two-Step Knockin of the $f h A$ and Site-Directed Mutagenesis of $f g k$}

Following the process of scarless-markerless knockout of $h s d M$ gene, all primers were in the Table 2 . The $\lambda$-red method was used to respectively insert the $\mathrm{Cm}^{R}$ gene into pseudogene $f h A$ in which 23 bp from 601 to 623 was lost, using primers $f h A-\lambda-\mathrm{F} / \mathrm{R}$, and into the $f g K$ gene in which a nucleotide GAA at 373 was changed to TAA to make a termination codon, using primers $f g K-\lambda-\mathrm{F} / \mathrm{R}$ in 


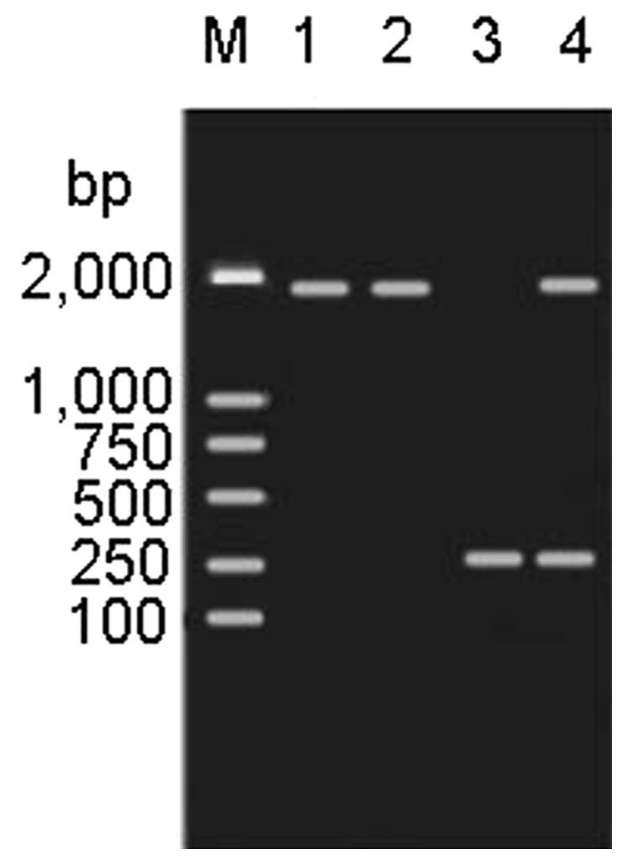

Fig. 3 PCR identification of $h s d M$ deletants from white colonies. M: DNA Marker DL2000; lane1: S06004 as a control; lane2: White colony 1(wild type S06004); lane3: White colony 2 (S06004 $\Delta h s d M)$; lane4: Blue colony(S06004(pGMB152- $\Delta h s d M)$ ).

S. pullorum. We then cloned a real gene from S. enteritidis P125109 to replace the pseudogene in S. pullorum to construct an in vitro-modified allele. Using the blue/white screen strategy in addition to counter selection screening ( $\operatorname{sac} B$ gene), the mutants with a knocked-in $f h A$ gene and mutated $f l g k$ gene were screened. The two genes were amplified by PCR and sequenced for further confirmation.

\section{Results and Discussion}

After construction of the recombinant suicide plasmid pGMB152- $\Delta h s d M / K m^{R}$ and conjugation, only $S$. pullorum $h s d M:: \mathrm{Cm}^{R}$ pGMB152- $\Delta h s d M / \mathrm{Km}^{R}$ grew as single-crossover recombinants on LB plates with the antibiotics (chloramphenicol, streptomycin, ampicillin, kanamycin) and without DAP, all colonies were blue (Fig. 1 left-hand panel).

When blue S. pullorum hsdM::Cm ${ }^{R}$ pGMB152- $\Delta h s d M /$ $K \mathrm{~m}^{R}$ was sub-cultured in liquid media with sucrose and without $\mathrm{NaCl}$, a few bacteria turned white, indicating that plasmid excision double-crossover had occurred (Fig. 1 right-hand panel).

The theoretical probability of finding deletants among the white colonies would be $50 \%$, because the two gene arms have an identical chance to exchange with the homologous sequence in the target genome. Approximately $10 \%$ of all white colonies were found to be deletants based on incomplete statistics. White deletants were confirmed by PCR with $\Delta h s d M$-YZ primers based on their amplicon size and antibiotic resistances; wild-type colonies were excluded (Fig. 2). The amplicon size should be $301 \mathrm{bp}$ for the white deletants, $1920 \mathrm{bp}$ for white wild-type strain, and both $1920 \mathrm{bp}$ and $301 \mathrm{bp}$ for the blue clones as a control. The amplicon sizes were consistent with the expected results.

Gene knockin and site-directed mutagenesis might be more difficult than gene knockout. We believed that improved plasmid could also be applied successfully for scarless-markerless gene knockin to repair the $f h B$ and flgK pseudogenes in $S$. pullorum, following the process summarized in Fig. 3. After repair, the two genes, $f h A$ and $f g K$, were amplified and sequenced for further confirmation. The results showed that the original pseudogenes were replaced by real genes. We successfully generated the knockin of the real $f h A$ gene using $23 \mathrm{bp}$ from 601 to 623 and performed site-directed mutagenesis of $\mathrm{fgK}$ to change nucleotide 373 from TAA, which is a termination codon, to GAA in S. pullorum (Fig. 4).

In this study, we found that sucrose-counter selection was relatively inefficient, as indicated by the presence of large numbers of blue colonies among the transformants (Fig. 1 right-hand panel) in S. pullorum, possibly because of host adaptation. This further confirmed that doublecrossover events occurred rarely in bacteria following the traditional method $[7,16]$. The results indicated that we could select random single-crossover recombinant bacteria without needing to reassess their sucrose sensitivity [8].

The larger size of vector pGMB152 might make molecular manipulations more difficult. To overcome this problem, an antibiotic resistance gene was cloned in one flank of the in vitro-modified allele for easy selection of recombinants, such as the kanamycin resistance gene in this study (Fig. 1).

This study is the first report of a suicide plasmid comprising the complete lac $Z$ gene that allows visual selection of double-crossover mutants. Although pGMB152 had only one modification, i.e., the entire lacZYA operon, compared with pGMB151, it was more efficient and less time consuming in producing allelic exchanges of gram-negative bacteria because of its color discrimination. Thus, the improved suicide plasmid, pGMB152, will be helpful for precise and scarless-markerless genome genetic modification. 
Fig. 4 The process of two-step scarless-markerless genome genetic modification based on suicide plasmid pGMB152

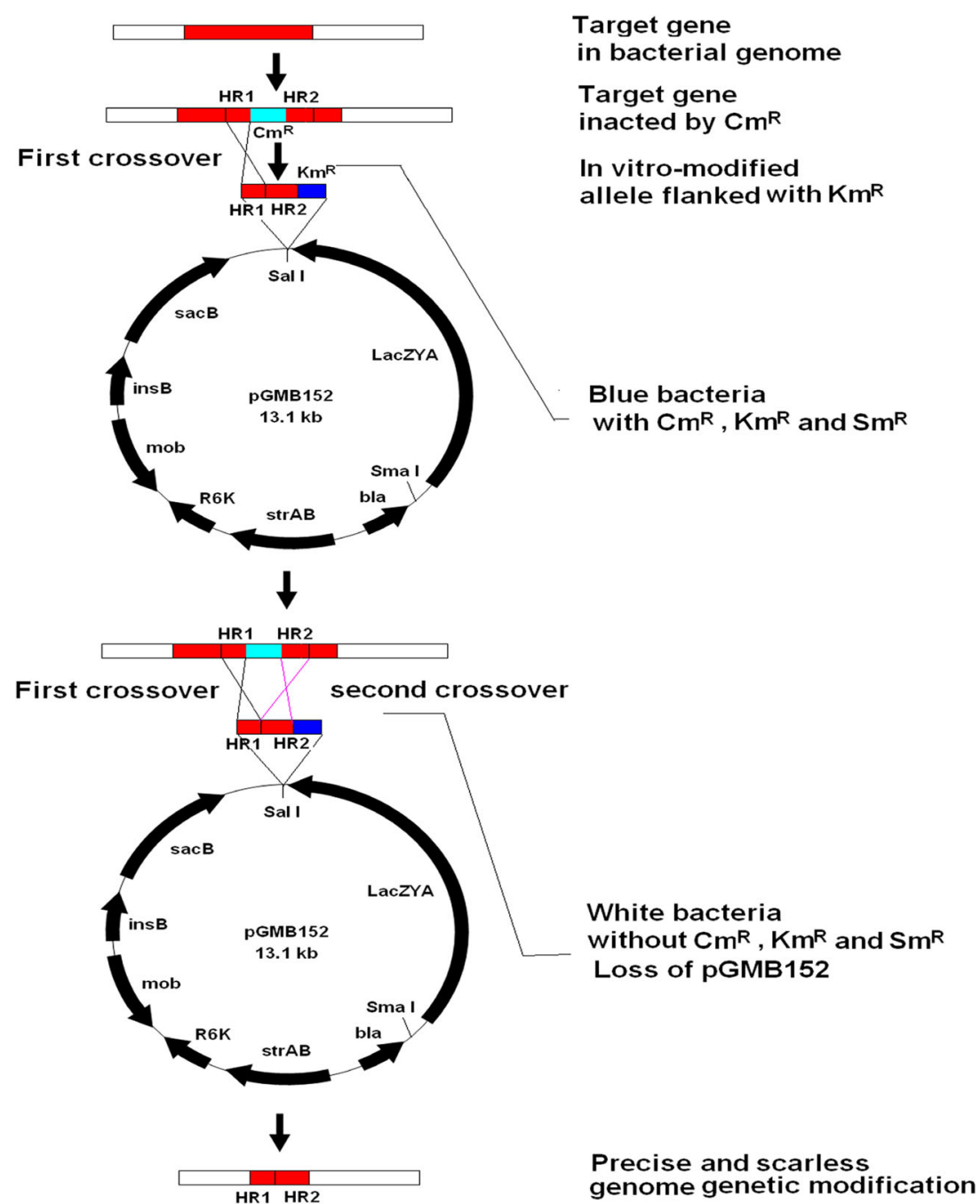

Acknowledgments We are grateful to Prof. Paul Barrow of the University of Nottingham for his help and suggestions during the writing and revision of this manuscript. This work was supported by key projects in the National Natural Science Foundation of China (31320103907, 31230070), the High Technology Research and Development of China (863 program, 2011AA10A210-2), the Natural Science Foundation of Jiangsu Province of China (BK20151306), and the Postdoctoral Science Foundation of China (2014M551670).

\section{Compliance with Ethical Standards}

Conflict of interest The authors declare that they have no competing interests.

Open Access This article is distributed under the terms of the Creative Commons Attribution 4.0 International License (http://crea tivecommons.org/licenses/by/4.0/), which permits unrestricted use, distribution, and reproduction in any medium, provided you give appropriate credit to the original author(s) and the source, provide a link to the Creative Commons license, and indicate if changes were made.

\section{References}

1. Bäumler AJ, Tsolis RM, Van der velden AWM, Stojiljkovic I, Anic S, Heffron F (1996) Identification of a new iron regulated locus of Salmonella typhi. Gene 183:207-213

2. Chaffin DO, Rubens CE (1998) Blue/white screening of recombinant plasmids in gram-positive bacteria by interruption of alkaline phosphatase gene (phoZ) expression. Gene 219:91-99

3. Datsenko KA, Wanner BL (2000) One-step inactivation of chromosomal genes in Escherichia coli $\mathrm{K}-12$ using PCR products. Proc Natl Acad Sci USA 97:6640-6645

4. Deng WY, Puente JL, Gruenheid S, Li YL, Vallance BA, Vázquez A, Barba J, Ibarra JA, O'Donnell P, Metalnikov P, Ashman K, Lee S, Goode D, Pawson T, Finlay BB (2004) Dissecting virulence: systematic and functional analyses of a pathogenicity island. Proc Natl Acad Sci USA 101:3597-3602

5. Forster BM, Bitar AP, Marquis H (2014) A non-catalytic histidine residue influences the function of the metalloprotease of Listeria monocytogenes. Microbiol 160:142-148

6. Fried L, Lassak J, Jung K (2012) A comprehensive toolbox for the rapid construction of lacZ fusion reporters. J Microbiol Meth 91:537-543 
7. Gay P, Lecoq D, Steinmetz M, Ferrari E, Hoch JA (1983) Cloning structural gene $s a c B$, which codes for exoenzyme levansucrase of Bacillus subtilis: expression of the gene in Escherichia coli. J Bacteriol 153:1424-1431

8. Geng SZ, Jiao XA, Pan ZM, Chen XJ, Zhang XM, Chen X (2009) An improved method to knock out the asd gene of Salmonella enterica serovar Pullorum. J Biomed Biotechnol. http://www. hindawi.com/journals/jbb/2009/646380.html

9. Geng SZ, Jiao XA, Barrow PA, Pan ZM, Chen X (2014) Virulence determinants of Salmonella Gallinarum biovar Pullorum identified by PCR signature-tagged mutagenesis and the spiC mutant as a candidate live attenuated vaccine. Vet Microbiol 168:388-394

10. Kang HY, Srinivasan J, Curtiss RIII (2002) Immune responses to recombinant pneumococcal PspA antigen delivered by live attenuated Salmonella enterica serovar Typhimurium vaccine. Infect Immun 70:1739-1749

11. Khan AQ, Zhao LC, Hirose K, Miyake M, Li TM, HashimotoY Kawamura Y, Ezaki T (1998) Salmonella typhi rpoS mutant is less cytotoxic than the parent strain but survives inside resting THP-1 macrophages. FEMS Microbiol Lett 161:201-208

12. McFadden J (1996) Recombination in mycobacteria. Mol Microbiol 21:205-211
13. Metcalf WW, Jiang W, Daniels LL, Kim SK, Haldimann A, Wanner BL (1996) Conditionally replicative and conjugative plasmids carrying lacZ $\alpha$ for cloning, mutagenesis, and allele replacement in bacteria. Plasmid 35:1-13

14. Navas J, González-Zorn B, Ladrón N, Garrido P, Vázquez-Boland JA (2001) Identification and mutagenesis by allelic exchange of choE, encoding a cholesterol oxidase from the intracellular pathogen Rhodococcusequi. J Bacteriol 183:4796-4805

15. Ortiz-Martín I, Macho AP, Lambersten L, Ramos C, Beuzón CR (2006) Suicide vectors for antibiotic marker exchange and rapid generation of multiple knockout mutants by allelic exchange in gram-negative bacteria. J Microbiol Meth 67:395-407

16. Philippe N, Alcaraz JP, Coursange E, Geiselmann J, Schneider D (2004) Improvement of pCVD442, a suicide plasmid for gene allele exchange in bacteria. Plasmid 51:246-255

17. Reyrat JM, Pelicic V, Gicquel B, Rappuoli R (1998) Counterselectable markers: untapped tools for bacterial genetics and pathogenesis. Infect Immun 66:4011-4017

18. Simon R, Priefer U, Puhler A (1983) A broad host range mobilisation system for in vivo genetic engineering: transposon mutagenesis in gram-negative bacteria. Nat Biotechnol $1: 784-791$ 